\title{
Biodegradación de espumas plásticas por larvas de insectos: ¿una estrategia sustentable?
}

\author{
Anlui Rodríguez-Carreón ${ }^{1}$, Yuridia Ortiz-Rivera ${ }^{1}$, \\ Claudia Carolina Hernández-Peña ${ }^{1}$ y Coyolxauhqui Figueroa ${ }^{2 *}$ \\ ${ }^{1}$ Programa de Biotecnología. Departamento de Ciencias Químico Biológicas, Instituto de Ciencias \\ Biomédicas, Universidad Autónoma de Ciudad Juárez. Av. Benjamín Franklin \#4650, Zona \\ PRONAF, ${ }^{2}$ Herbario UACJ. Programa de Biología, Ciudad Juárez, 32310, Chihuahua, México. \\ E-mail: *cfigueroa@uacj.mx
}

\begin{abstract}
RESUMEN
En la actualidad, la mayoría de las actividades humanas dependen en gran medida del uso de los plásticos, cuya producción y consumo han aumentado considerablemente en las últimas décadas. La utilización de productos plásticos ha tenido impactos positivos para nuestro bienestar; sin embargo, los efectos negativos derivados de su disposición inapropiada son motivo de gran preocupación, especialmente durante la actual emergencia sanitaria provocada por la COVID-19, en la que el uso de plásticos por una ocasión ha aumentado drásticamente. A pesar de que algunos polímeros plásticos como el polietileno (PE) y el poliestireno (PS) pueden reciclarse, la alta demanda energética y tecnológica, el empleo de sustancias potencialmente tóxicas y la baja rentabilidad económica del proceso, vuelven a esta opción poco viable. Ante esta situación, el tratamiento biológico de plásticos a través de organismos degradadores del PE y del PS, como las larvas de la harina y de la cera, surge como una alternativa prometedora y ha llamado la atención de investigadores alrededor del mundo. El objetivo de esta revisión es sintetizar los avances más recientes en el campo de la biodegradación de plásticos por larvas de insectos y un análisis de las tendencias actuales y futuras en esta creciente área de investigación.
\end{abstract}

Palabras clave: biodegradación, polietileno, poliestireno, Pyralideae, Tenebrionideae.

\section{Biodegradation of plastic foams by insect larvae: a sustainable strategy?}

\begin{abstract}
Many modern human activities rely greatly on plastic foams, whose consumption and production have considerably increased in the past decades. The use of plastic products has had positive impacts on human general welfare; however, the negative effects derived from their inappropriate disposition have become a major concern, especially during the current COVID-19 sanitary emergency, in which the utilization of single-use plastics has rapidly increased. Although some plastic polymers such as polyethylene (PE) and polystyrene (PS) can be recycled, the high demand for energy and technology, the use of potentially toxic substances and the low profitability of the process, make this option nonoptimal. In this situation, biological treatment of plastic waste is emerging as a promising alternative and the use of PE and PS-eating organisms, such as mealworms and waxworms, has attracted the attention of researchers around the world. The aim of this review is to synthesize the most recent advances in the field of plastics polymers degradation by insects larvae and an analysis of the current and future trends of this developing research area.
\end{abstract}

Keywords: biodegradation, polyethylene, polystyrene, Pyralideae, Tenebrionideae. 


\section{INTRODUCCIÓN}

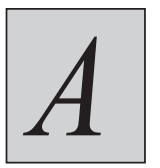

ctualmente las actividades humanas dependen en gran parte del uso de productos plásticos, cuyo consumo y producción ha aumentado en las últimas décadas (Geyer, Jambeck \& Law, 2017); en particular, con la actual emergencia sanitaria provocada por la COVID-19 (ONU, 2020). El empleo de productos plásticos ha tenido un impacto positivo en el bienestar humano y actualmente son imprescindibles para mantener las normas de higiene; sin embargo, también producen un efecto negativo derivado del deficiente manejo de estos residuos, afectando especialmente la salud de los ecosistemas (Eriksen et al., 2014). En el medio ambiente, los restos de espumas de polietileno (PE) y poliestireno (PS) pueden ser confundidos con alimento y ser ingeridos por la fauna silvestre, causándole enfermedades y hasta la muerte. Además, algunos de estos plásticos lixivian toxinas, que pueden bio-acumularse, por ejemplo, en los tejidos de pescados y mariscos (Fabreag \& Familara, 2019). La preocupación ambiental por la contaminación de las espumas plásticas gira en torno a cuatro elementos fundamentales: (I) su lenta degradación; (II) la producción de residuos; (III) su fuente de generación es el petróleo considerado materia prima no renovable y (IV) algunos de los insumos químicos utilizados para producirlos son nocivos para el ambiente (Arthuz-López \& Pérez-Mora, 2019).

Los plásticos han aumentado su presencia en los residuos domésticos y se ha estimado que en conjunto el plástico rígido y el de película, poliestireno expandido y el de polietileno constituyen el $11.67 \%$ del total de residuos sólidos urbanos en México (Vázquez-Morillas et al., 2018), mientras las estimaciones a nivel mundial indican que solo el 9\% se recicla (ONU, 2020). Si bien tanto el PE como el PS se consideran reciclables, los mecanismos actuales para este procesamiento distan de ser los ideales debido a la alta demanda energética y tecnológica, la utilización de químicos tóxicos y la baja rentabilidad económica derivadas de su transformación (Maharana, Negi \& Mohanty, 2007). Ante esta situación, la biodegradación de plásticos parece ser una opción prometedora para el tratamiento de este tipo de desechos. Recientemente, se ha demostrado la capacidad de diversos organismos superiores para biodegradar plásticos, como las larvas de palomillas (Galleria mellonella L.: Bombelli, Howe \& Bertocchini, 2017; Achroia grisella F.: Kundungal, Gangarapu, Sarangapani, Patchaiyappan \& Purushothaman, 2019 a, b) y las larvas de escarabajos (Familia: Tenebronidae, Yang, Wu, Zhao \& Jiang, 2014, Yang et al., 2015 a, b, 2018 a, b, Yang, Wang \& Xia, 2020). Estos hallazgos han permitido replantear preguntas acerca de la factibilidad de la biodegradación de plásticos y han establecido una novedosa perspectiva para la investigación de nuevos organismos que podrían presentar estas características. Esta revisión pretende resumir y analizar la información que se ha generado hasta el momento acerca de las especies que son capaces de biodegradar polímeros plásticos y comparar los diferentes datos de degradación generados previamente, con la finalidad de responder si la biodegradación de espumas plásticas por larvas de insectos representa una alternativa viable para contrarrestar la contaminación generada por estos materiales.

\section{BIOdEGRAdACIÓN DE PLÁSTICOS POR LARVAS DE LA HARINA (COLEOPTERA: TENEBRIONIDAE)}

Se les conoce con el nombre común de gusanos de la harina a las larvas de las especies de escarabajos de la familia Tenebrionidae. De esta, cuatro especies destacan por su capacidad para consumir polímeros plásticos: las del escarabajo amarillo (Tenebrio molitor L.), del escarabajo negro (Tenebrio obscurus F.), del escarabajo castaño (Tribolium castaneum H.) y las del escarabajo gigante (Zophobas atratus F.), (Tablas I y II). El desarrollo de estos organismos se da en cuatro etapas o estadios: huevo, larva, pupa y adulto. Las larvas del género Tenebrio típicamente miden entre 2.5 y $3.5 \mathrm{~cm}$ de longitud (Bryning, Chambers \& Wakefield, 2005), mientras que la longitud de las del género Zophobas oscilan entre los 4 y $6 \mathrm{~cm}$ (Leung et al., 2012); las más pequeñas son las del género Tribolium con apenas $6 \mathrm{~mm}$. Estas especies son originarias de Europa y actualmente se encuentran distribuidas a escala global.

Yang et al. (2015 a) mostraron las primeras evidencias sobre la degradación de espumas plásticas por larvas de insectos cuando reportaron que las de T. molitor fueron capaces de digerir poliestireno expandido (EPS), logrando una degradación eficiente del material dentro de su tracto intestinal en un tiempo de retención menor a $24 \mathrm{~h}$. Durante esta investigación no se detectaron diferencias significativas entre la tasa de supervivencia de las larvas alimentadas con una dieta convencional y aquellas alimentadas exclusivamente con EPS y basándose en el uso de sustratos marcados con $\alpha^{13} \mathrm{C}$ y $\beta^{13} \mathrm{C}$, los autores detectaron que el $47.7 \%$ del carbono del EPS ingerido fue mineralizado y convertido en $\mathrm{CO}_{2}$, mientras que el $49.2 \%$ fue excretado como humus y alrededor del $1 \%$ se incorporó como biomasa.

Derivado de estos estudios, también fue analizado el papel de la microbiota intestinal en la degradación del EPS (Yang et al., 2015 b), lográndose el aislamiento y la identificación de una cepa bacteriana (Exiguobacterium sp. YT2) que fue capaz de formar una biopelícula sobre la superficie del EPS en un periodo de 28 días y generar agrietamientos visibles $(0.2-0.3 \mathrm{~mm}$ de diámetro) en el material plástico, disminuyendo su peso molecular y aumentando su solubilidad (Tabla III). 
Tabla I. Degradación de PS por larvas de la harina.

\begin{tabular}{|c|c|c|c|c|c|c|c|}
\hline Organismos & $\begin{array}{l}\text { Tipos de } \\
\text { PS }\end{array}$ & Tiempo (d) & $\begin{array}{c}\text { Pérdida de } \\
\text { masa }(\%)\end{array}$ & $\begin{array}{l}\operatorname{EED}(\mathbf{m g} \\
\left.\operatorname{larva}^{-1} \mathbf{d}^{-1}\right)\end{array}$ & $\begin{array}{c}\text { Peso } \\
\text { molecular }\end{array}$ & $\begin{array}{l}\text { Modificaciones } \\
\text { estructurales }\end{array}$ & Referencias \\
\hline T. molitor & EPS & 30 & $31 \pm 1.7$ & 0.12 & Disminuyó & Detectadas & $\begin{array}{l}\text { Yang et al., } \\
2015 \mathrm{a}\end{array}$ \\
\hline T. molitor & EPS & 32 & $45.8 \pm 1.9$ & 0.22 & Disminuyó & Detectadas & $\begin{array}{l}\text { Yang et al., } \\
2018 \text { a }\end{array}$ \\
\hline T. molitor & EPS & 32 & $\mathrm{NE}$ & $\begin{array}{l}0.17 \pm \\
0.03^{\mathrm{a}}\end{array}$ & Disminuyó & Detectadas & $\begin{array}{l}\text { Yang et al., } \\
2018 \text { b }\end{array}$ \\
\hline T. molitor & EPS & 91 & $16.7 \pm 0.2$ & $\mathrm{NE}$ & $\mathrm{NE}$ & $\mathrm{NE}$ & $\begin{array}{l}\text { Matyja et al., } \\
2020\end{array}$ \\
\hline T. castaneum & EPS & 20 & 7.14 & $0.83^{c}$ & $\mathrm{NE}$ & $\mathrm{NE}$ & $\begin{array}{l}\text { Fabreag \& } \\
\text { Familara, } 2019\end{array}$ \\
\hline Z. atratus & EPS & 28 & $65 \pm 6.6$ & 0.58 & Disminuyó & Detectadas & $\begin{array}{l}\text { Yang et al., } \\
2020\end{array}$ \\
\hline Z. atratus & EPS & 7 & 21 & $\mathrm{NE}$ & $\mathrm{NE}$ & $\mathrm{NE}$ & $\begin{array}{l}\text { Kim et al., } \\
2020\end{array}$ \\
\hline
\end{tabular}

${ }^{a}$ Datos obtenidos de 6 ubicaciones geográficas distintas ( 5 en USA, 1 en Irlanda del norte) ${ }^{\text {b }}$ Promedio obtenido de 3 tratamientos con larvas provenientes de 3 regiones geográficas distintas. ${ }^{\mathrm{C}}$ fueron utilizados adultos. EPS: Poliestireno expandido. XPS: Poliestireno extruido. EED: Eficiencia específica de degradación. NE: No especificado.

Tabla II. Degradación de PE por larvas de la harina.

\begin{tabular}{|c|c|c|c|c|c|c|c|}
\hline Organismos & Tipos de PE & $\begin{array}{l}\text { Tiempo de } \\
\text { tratamiento } \\
\text { (d) }\end{array}$ & $\begin{array}{l}\text { Pérdida de } \\
\text { masa }(\%)\end{array}$ & $\begin{array}{c}\text { EED } \\
\left(\mathrm{mg} \mathrm{larva}^{-1}\right. \\
\left.\mathrm{d}^{-1}\right)\end{array}$ & $\begin{array}{c}\text { Peso } \\
\text { molecular }\end{array}$ & $\begin{array}{c}\text { Modificaciones } \\
\text { estructurales }\end{array}$ & Referencias \\
\hline T. molitor & PE granulado & 70 & 16.6 & $\mathrm{NE}$ & $\mathrm{NE}$ & $\mathrm{NE}$ & $\begin{array}{l}\text { Przemieniecki et } \\
\text { al., } 2020\end{array}$ \\
\hline T. molitor & $\begin{array}{l}\text { Láminas de } \\
\text { LDPE }\end{array}$ & 38 & 3.5 & 0.023 & $\mathrm{NE}$ & $\mathrm{NE}$ & $\begin{array}{l}\text { Billen et al., } \\
2020\end{array}$ \\
\hline T. molitor & LDPE & 32 & $45 \pm 1.2$ & 0.22 & Disminuyó & Detectadas & $\begin{array}{l}\text { Brandon et al., } \\
2018\end{array}$ \\
\hline T. molitor & $\begin{array}{c}\text { Partículas de } \\
\text { PE }\end{array}$ & 32 & $29.5 \pm 7.4^{\mathrm{a}}$ & 0.12 & Disminuyó & Detectadas & Wu et al., 2019 \\
\hline Z. atratus & $\begin{array}{l}\text { Gránulos de } \\
\text { LDPE }\end{array}$ & 33 & 35 & 0.58 & Aumentó & Detectadas & Peng et al., 2020 \\
\hline
\end{tabular}

PE: Polietileno. LDPE: Polietileno de baja densidad. EED: Eficiencia específica de degradación. NE: No especificado. 
Tabla III. Degradación de plásticos por bacterias entéricas

\begin{tabular}{|l|c|c|c|c|c|l|}
\hline $\begin{array}{c}\text { Organismos (lugar de } \\
\text { aislamiento) }\end{array}$ & $\begin{array}{c}\text { Tipos de } \\
\text { polímeros }\end{array}$ & $\begin{array}{c}\text { Tiempo de } \\
\text { tratamiento } \\
\mathbf{( d )}\end{array}$ & $\begin{array}{c}\text { Pérdida } \\
\mathbf{d e} \text { masa } \\
\mathbf{( \% )}\end{array}$ & $\begin{array}{c}\text { Peso } \\
\text { molecular }\end{array}$ & $\begin{array}{c}\text { Modificaciones } \\
\text { estructurales }\end{array}$ & Referencias \\
\hline $\begin{array}{l}\text { Enterobacter absuriae } \\
(\text { P. } \text { interpunctella })\end{array}$ & LDPE & 60 & $6.1 \pm 0.3$ & Disminuyó & Detectadas & $\begin{array}{l}\text { Yang } \text { et al., } \\
2014\end{array}$ \\
\hline $\begin{array}{l}\text { Bacillus } \text { sp. } \\
(P . \text { interpunctella })\end{array}$ & LDPE & 60 & $10.7 \pm 0.2$ & Disminuyó & Detectadas & $\begin{array}{l}\text { Yang } \text { et al., } \\
2014\end{array}$ \\
\hline $\begin{array}{l}\text { Exiguobacterium sp.YT2 } \\
(T \text {. molitor })\end{array}$ & EPS & 60 & $7.4 \pm 0.4$ & Disminuyó & Detectadas & $\begin{array}{l}\text { Yang } \text { et al., } \\
2015 \mathrm{~b}\end{array}$ \\
\hline $\begin{array}{l}\text { Acinetobacter } \text { sp. AnTc-1 } \\
(T . \text { castaneum })\end{array}$ & $\begin{array}{c}\text { PS Estándar } \\
\text { (CAS 33,165-1) }\end{array}$ & 60 & $12.14 \pm 1.4$ & Disminuyó & Detectadas & $\begin{array}{l}\text { Wang } \text { et al., } \\
2020\end{array}$ \\
\hline
\end{tabular}

LDPE: Polietileno de baja densidad. EPS: Poliestireno expandido. PS: Poliestireno.

Un estudio realizado por otro grupo de investigación (Yang et al., 2018 a) evaluó la influencia de la temperatura, la composición de la dieta y la densidad del plástico en la eficiencia de degradación del PS en larvas comerciales de T. molitor provenientes de Estados Unidos. Los resultados de esta investigación concluyeron que cuando las larvas fueron cultivadas con una dieta mixta de trigo/PS, la eficiencia de degradación aumentó hasta en un $72 \%$ con respecto a las larvas alimentadas exclusivamente con PS. La temperatura tuvo una influencia significativa en el proceso de degradación, encontrándose un valor óptimo de $25^{\circ} \mathrm{C}$. Además, los mismos autores observaron que las larvas tuvieron una mayor eficiencia de degradación del PS de baja densidad en comparación con el de alta densidad. Se ha confirmado que T. molitor tiene la capacidad para degradar PS, como una característica intrínseca y conservada en la mayoría de las variedades de esta especie (Yang et al., 2018 b).

Nukmal, Umar, Amanda \& Kanedi, (2018) compararon las tasas de supervivencia, las ganancias de masa en sus distintos estadios, los periodos de pupación y la ovoposición de T. molitor cultivados con dietas convencionales (almidón de yuca) y dietas a base de EPS y poliestireno extruido (XPS). Los resultados mostraron que, en comparación con la dieta convencional, las dietas exclusivas de EPS y XPS no aumentaron significativamente la tasa de mortalidad de las larvas. Ambos tipos de PS promovieron periodos de pupación significativamente mayores y disminuyeron la ovoposición de los imagos al reducirse el número de huevecillos en los cultivos. Sólo aquellos insectos cultivados con XPS desarrollaron menor masa en las larvas, pupas e imagos.

Brandon et al. (2018) estudiaron la biodegradación de una mezcla de LDPE (polietileno de baja densidad) y EPS por larvas de T. molitor. Los resultados obtenidos muestran que las larvas fueron capaces de mineralizar LDPE a tasas similares a las reportadas durante la degradación de EPS. De manera interesante, los estudios de cromatografía de permeación en gel (GPC) indicaron que el peso molecular del LDPE excretado disminuyó en $40.1 \pm 8.5 \%$ mientras que el peso molecular del EPS disminuyó $12.8 \pm 3.1 \%$, además, los análisis de resonancia magnética nuclear (NMR) y espectroscopía infrarroja (FTIR) revelaron modificaciones químicas atribuibles a una oxidación parcial del polímero. La disminución en el peso molecular y la oxidación de las cadenas hidrocarbonadas de un compuesto suelen estar directamente relacionadas con un aumento en la biodegradabilidad del mismo. Aunado a lo anterior, los autores analizaron el microbioma intestinal de las larvas mediante secuenciación genética de segunda generación durante la biodegradación de ambos polímeros, encontrando que las unidades taxonómicas operables Citrobacter sp. y Kosakonia sp. estuvieron asociadas con el consumo de LDPE y EPS respectivamente.

En el trabajo realizado por Peng et al. (2019) se estudió la capacidad de las larvas de los escarabajos T. obscurus y T. molitor para degradar EPS. Los resultados mostraron que las larvas de T. obscurus exhibieron una eficiencia de degradación del $40 \%$ mayor que la de T. molitor. Los análisis de FTIR y termogravimetría (TGA) del material excretado por las larvas de ambas especies indicaron una disminución significativa del peso molecular del polímero, así como la formación de grupos funcionales hidrofílicos, lo que generaría una mayor solubilidad de la sustancia y, comúnmente, una disminución en su toxicidad y recalcitrancia. Por último, los autores reportaron que la tasa de degradación de EPS tuvo un incremento importante en ambas especies cuando su dieta fue enriquecida con harina de maíz y salvado de trigo, esto parece indicar que, a pesar de contar con un sustrato capaz de abastecer sus demandas energéticas, la voracidad de los organismos por el polímero plástico no decrece e incluso podría promoverse. 
Otra investigación reciente ha explorado la capacidad de T. molitor para degradar otros polímeros plásticos recalcitrantes como caucho vulcanizado y viruta de neumáticos (Aboelkheir, Visconte, Oliveira, Toledo-Filho \& Souza, 2019). Los resultados de esta investigación muestran que el contacto con las larvas después de 30 días generó modificaciones químicas y estructurales en ambos materiales; sin embargo, los niveles de degradación, evaluados por la pérdida de masa de los materiales, fueron bajos $(3.15 \%$ para el caucho vulcanizado, $1.7 \%$ para la viruta de neumático).

El potencial tratamiento de microplásticos de policloruro de vinilo (PVC), PE y PS por larvas de T. molitor también ha sido investigado (Wu, Tao \& Wong, 2019), durante este estudio, se observó que 3 grupos de larvas provenientes de 3 provincias diferentes en China lograron ingerir los tres tipos de microplásticos; sin embargo, las dietas no suministraron energía suficiente para el crecimiento de los organismos. De los tres microplásticos analizados, el PVC se metabolizó en menor medida.

Otro reporte reciente (Xia, Wang, Huo \& Yang, 2020) mencionó la identificación de una nueva especie bacteriana, Mixta tenebrionis aislada del tracto intestinal de larvas de T. molitor que mostraron capacidad para degradar PS; sin embargo, no se especificó si este microorganismo es capaz de degradar el polímero plástico. En otro estudio se aisló una bacteria: Acinetobacter sp. AnTc-1 del tracto intestinal de larvas de $T$. castaneum capaces de masticar y digerir XPS (Wang, Xin, Shi \& Zhang, 2020). Los experimentos de NMR, TGA, GPC y microscopía electrónica (SEM y TEM) confirmaron la presencia de modificaciones estructurales, la formación de grupos funcionales y la disminución en el peso molecular del PS después de un periodo de 20 días de incubación de la cepa AnTc-1. Lo anterior sugiere que este microorganismo tiene la capacidad para degradar PS y posiblemente juega un papel importante en el metabolismo intestinal de $T$. castaneum.

Algunos autores han analizado los cambios en el microbioma y el perfil enzimático intestinal de T. molitor durante su desarrollo con una dieta basada en espuma de PE y otros plásticos (Przemieniecki, Kosewska, Ciesielski \& Kosewska, 2020). Sus resultados han sugerido que las enzimas esterasa y aril-amidasa podrían estar estrechamente relacionadas con la degradación de la espuma del PE, aunque la ruta exacta de la biotransformación de este polímero es aún incierta.

Recientemente se ha observado que la capacidad para degradar polímeros plásticos por escarabajos va más allá del género Tenebrio. Los adultos del escarabajo Tribolium castaneum han mostrado la capacidad para producir daños estructurales en recipientes de PE (Hassan, Qasim, Iqbal \& Jamil, 2014; Hassan et al., 2016) y en bloques de EPS, donde se pudo observar una pérdida significativa en la masa del material $(21.07 \%)$ durante un periodo de incubación de 20 días (Fabreag \& Familara, 2019). Sin embargo, el grado de despolimerización y las modificaciones estructurales (de haberlas) en las espumas plásticas no fueron confirmados mediante análisis instrumentales.

Por otro lado, en estudios realizados por Yang, Wang \& Xia (2020) con larvas del escarabajo rey Zophobas atratus $[=Z$. morio $]$ se observó que este insecto también posee una capacidad importante para metabolizar EPS en su tracto digestivo. Los autores reportaron una tasa específica de degradación de EPS hasta 4 veces mayor a las reportadas anteriormente en $T$. molitor. Además, los análisis respirométricos exhibieron una mineralización del $37.6 \%$ del EPS después de 16 días de tratamiento, tiempo durante el cual las larvas pudieron subsistir con una dieta exclusiva de material plástico. El análisis del humus generado durante el tratamiento sugiere que existe una despolimerización parcial de las cadenas largas del EPS dentro del tracto intestinal de las larvas con la consecuente formación de productos de bajo peso molecular, confirmándose la biotransformación de este material. Estos resultados aumentan la probabilidad de que la capacidad para biodegradar polímeros plásticos se extienda a otros miembros de la familia Tenebrionidae (una de las más grandes del orden Coleoptera: 20,000 especies; CifuentesRuiz \& Zaragoza-Caballero, 2014).

Otro grupo de investigación ha reportado que la cepa bacteriana Pseudomonas sp., aislada del tracto intestinal de Z. atratus ha exhibido capacidad para degradar PS. Mediante análisis de expresión génica por PCR cuantitativa reversa (RT- qPCR), se observó que algunas enzimas del tipo hidrolasas (serina-hidrolasa y s-formilglutatión hidrolasa) aumentaron considerablemente su expresión, mientras que otras hidrolasas y esterasas (alfa-beta hidrolasa, arilesterasa, tioesterasa) disminuyeron de manera importante su expresión (Kim et al., 2020). Estos resultados parecen indicar que una disminución de la actividad de ciertas enzimas, combinado con el incremento de otras es necesario para lograr una degradación eficiente del PS; sin embargo, aún se requiere de mayor investigación para comprender con más profundidad los mecanismos bioquímicos de la degradación de este polímero plástico.

\section{Biodegradación De PLÁSTICOS POR LARVAS DE LA CERA}

Los gusanos de la cera son las larvas de los insectos comúnmente conocidos como polillas de la cera. Entre estos organismos, tres especies han sido estudiadas por su capacidad para degradar plásticos: Achroia grisella F., Galleria mellonella L. y Plodia interpunctella H. (familia Pyralidae) (Tabla IV). Las larvas de la cera son plagas importantes y agresivas de las colmenas de abejas, éstas 
Tabla IV. Degradación de polímeros plásticos por larvas de la cera.

\begin{tabular}{|c|c|c|c|c|c|c|c|}
\hline Organismos & $\begin{array}{c}\text { Tipos de } \\
\text { PE } \\
\end{array}$ & Tiempo (d) & $\begin{array}{l}\text { Pérdida de } \\
\text { masa }(\%)\end{array}$ & $\begin{array}{c}\text { EED }(\mathbf{m g} \\
\left.\operatorname{larva}^{-1} \mathbf{d}^{-1}\right)\end{array}$ & $\begin{array}{c}\text { Peso } \\
\text { molecular }\end{array}$ & $\begin{array}{c}\text { Modificaciones } \\
\text { estructurales }\end{array}$ & Referencias \\
\hline P. interpunctella & LDPE & 28 & $31 \pm 1.7$ & 0.38 & Disminuyó & Detectadas & $\begin{array}{l}\text { Yang et al., } \\
2014\end{array}$ \\
\hline G. mellonella & LDPE & 0.5 & 13 & 0.23 & Disminuyó & Detectadas & $\begin{array}{l}\text { Bombelli et al., } \\
2017\end{array}$ \\
\hline A. grisella & $\begin{array}{l}\text { HDPE } \\
\text { PE }\end{array}$ & 8 & $43.3 \pm 1.6$ & 1.83 & Disminuyó & Detectadas & $\begin{array}{l}\text { Kundungal et } \\
\text { al., } 2019 \text { a }\end{array}$ \\
\hline G. mellonella & LDPE & 10 & $18.57 \pm 1.8$ & $\mathrm{NE}$ & Disminuyó & Detectadas & $\begin{array}{l}\text { Kundungal et } \\
\text { al., } 2019 \mathrm{~b}\end{array}$ \\
\hline G. mellonella & $\begin{array}{l}\text { LDPE y } \\
\text { EPS }\end{array}$ & 21 & $\mathrm{NE}$ & $\begin{array}{l}\text { LDPE: } 0.62 \\
\text { PS: } 0.28\end{array}$ & $\begin{array}{c}\text { LDPE: } \\
\text { Disminuyó } \\
\text { PS: Aumentó }\end{array}$ & Detectadas & Lou et al., 2020 \\
\hline
\end{tabular}

LDPE: Polietileno de baja densidad. HDPE: Polietileno de alta densidad. EPS: Poliestireno expandido. EED: Eficiencia específica de degradación. NE: No especificado.

viven como parásitos y se alimentan de la cera, de ahí su nombre. Las larvas de la cera, al igual que las de la harina, descritas en la sección anterior, se usan extensivamente como alimento para mascotas, como reptiles, anfibios, aves y también como carnadas para la pesca. A. grisella (polilla menor) y G. mellonella (polilla mayor), son dos especies cercanas que se producen comercialmente; por el contrario, P. interpunctella, llamada comúnmente polilla de la India, no es producida a gran escala.

Uno de los primeros reportes sobre biodegradación de plásticos por este tipo de organismos fue el de Yang, Yang, Wu, Zhao \& Jiang (2014), ellos encontraron que las larvas de la cera de la India ( $P$. interpunctella) eran capaces de masticar y comer películas de PE. A partir de los intestinos de la palomilla $P$. interpuctella, se han aislado dos líneas de bacterias, Enterobacter asburiae y Bacillus sp., ambas han mostrado la capacidad de descomponer PE en condiciones de laboratorio. En una prueba durante 28 días de incubación con estas bacterias, se observó disminución en la hidrofobicidad del plástico. Además, por medio de SEM y microscopía de fuerza atómica se observaron huecos y cavidades de 0.3$0.4 \mu \mathrm{m}$ de profundidad en el material.

Posteriormente, Bombelli et al. (2017) documentaron que cien larvas de G. mellonella consumieron casi $0.1 \mathrm{~g}$ de bolsas plásticas (LDPE) en 12 horas. La cera de las abejas está constituida por lípidos compuestos, incluyendo alquenos, alcanos, ésteres y ácidos grasos. El hidrocarburo más frecuentemente presente en la cera de abeja es el etileno $\left(\mathrm{CH}_{2}-\mathrm{CH}_{2}\right)$, por esta razón, los autores hipotetizaron que las larvas de estos insectos son capaces de alimentarse del PE. Este trabajo sirvió como base para el desarrollo de una nueva área de investigación enfocada en la biodegradación de plásticos por larvas de la cera.

En el año 2019, se publicó evidencia de la degradación del polietileno de alta densidad (HDPE) por la polilla menor de la cera A. grisella (Kundungal et al., 2019 a). Los resultados obtenidos mostraron que, en un periodo de ocho días, el consumo de HDPE aumentó, llegando a un nivel de ingesta de $1.83 \mathrm{mg}$ día $^{-1}$ larva $^{-1}$. Una dieta combinada (cera de abeja y PE) aumentó el consumo de HDPE y fue necesaria para que el insecto culminara su ciclo de vida y tuviera una segunda generación de larvas degradadoras de plástico. A su vez, el mismo equipo de colaboradores evaluó el efecto del pretratamiento con radiación solar sobre LDPE que posteriormente fue consumido por A. grisella. El pretratamiento consistió en la exposición a la luz solar de 8.00 a.m. a 4.00 p.m. por 15 días (Kundungal et al., 2019 b). El plástico irradiado se agrietó y esto favoreció la voracidad de las larvas, así como un aumento en la mineralización de las excretas, lo que indicó una mayor biodegradación del material.

Otros estudios se han enfocado en tratar de comprender mejor las rutas de biodegradación de los plásticos. Kong et al. (2019) observaron que la cera de abeja fue parcialmente degradada por larvas de G. melonella cuya microbiota intestinal fue inhibida por antibióticos, indicando que las largas cadenas de hidrocarburos son inicialmente despolimerizadas por el hospedero y liberan las largas cadenas de ácidos grasos que subsecuentemente serán metabolizadas por la microbiota del estómago. Cuando G. melonella fue alimentada con cera de abeja hubo un aumento de transcriptos que codifican para carboxilesterasas específicas, lipasas y enzimas involucradas en la oxidación de ácidos grasos. A pesar de estos hallazgos, 
aún no se conocen con detalle las rutas enzimáticas de la degradación del PE; sin embargo, Kong et al. (2019) apoyaron la propuesta de Bombelli et al. (2017), en la que se sugirió que las rutas enzimáticas involucradas en la degradación del plástico y la cera de abeja son similares.

Durante el transcurso del año 2020, Cassone, Grove, Elebute, Villanueva \& LeMoine presentaron evidencia de una relación entre el microbioma intestinal de las larvas de G. melonella, la biodegradación de LDPE y la producción de etilenglicol como producto metabólico. Ellos confirmaron bioquímicamente que las larvas consumen y metabolizan LDPE. Además, estos autores identificaron que el género de bacterias Acinetobacter está directamente involucrado en la biodegradación del PE. También encontraron que el microbioma del estómago de estos lepidópteros está influenciado por factores ambientales, la etapa del desarrollo larval y el tipo de dieta.

También en el año 2020, Lou y colaboradores evaluaron la degradación del PE y el PS por las larvas de G. melonella y el efecto de una dieta complementaria en el consumo de estos polímeros. Ellos observaron pérdidas significativas de masa en los plásticos en un periodo de 21 días, con la subsecuente formación de ácidos grasos y ésteres de cadena larga en la fracción intestinal de las muestras, además de una disminución de los compuestos aromáticos complejos en el humus de las larvas, lo que sugiere una biodegradación parcial de los materiales. El análisis del microbioma intestinal reveló que Bacillus y Serratia estuvieron significativamente asociadas con la dieta de PE y PS respectivamente. La suplementación con cera de abeja y salvado de trigo incrementó las tasas de sobrevivencia de las larvas, incentivó una mayor diversidad microbiana y aumentó el grado de despolimerización de los materiales, pero disminuyó el consumo de plástico en comparación con las larvas que se alimentaron solo con PE o PS. Este hallazgo contrasta con otros que han sido reportados para larvas de escarabajos (Yang et al., 2018 a; Peng et al., 2019) y para $A$. grisella (Kundungal et al., 2019 a), donde se observaron aumentos significativos en las tasas de consumo de los plásticos cuando los organismos se cultivaron con una dieta complementaria. Esto parece indicar que la promoción en el consumo de plásticos por efecto de una dieta enriquecida es una característica que dependerá de la especie utilizada, por lo que resulta imprescindible el desarrollo de más investigación en esta área, que se enfoque en el estudio individual de las especies y en la optimización de los parámetros de cultivo, con el fin de maximizar la capacidad de degradación de los polímeros por estos sistemas biológicos.

Finalmente, Peydaei, Bagheri, Gurevich, de Jonge \& Nielsen (2020) evaluaron el papel de las glándulas salivales de G. mellonella en la degradación del PE. Durante este trabajo se analizaron los cambios en la superficie del PE que fue masticado y consumido, observándose la formación de cavidades e intermediarios de degradación -incluyendo grupos carbonilo- en el material plástico. Lo anterior indicó que la saliva producida por este insecto puede asistir en la degradación del PE. Posterior a ello, se investigaron también los efectos bioquímicos de la exposición al PE sobre la composición del proteoma de las glándulas salivales. Las proteínas cuya expresión fue significativamente afectada por la exposición al PE evidenciaron la inducción de rutas enzimáticas asociadas con la beta-oxidación de ácidos grasos. Los resultados obtenidos en este estudio revelaron que durante la masticación de PE por G. mellonella se forman, de manera simultánea, "picaduras" a lo largo de los bordes de los agujeros producidos por las larvas y productos de degradación que químicamente podrían parecerse a los ácidos grasos. Estos productos de degradación también fueron detectados en suspensiones del PE con saliva de los insectos, lo que reveló que las glándulas salivales podrían estar involucradas en la degradación del plástico desde la misma masticación del material. La exposición a PE no solo promovió una mayor síntesis de proteínas asociadas a la $\beta$-oxidación de ácidos grasos, sino que también indujo la producción de estructuras similares a la seda en el borde de los agujeros producidos por las larvas y una expresión significativamente mayor de fibrohexamerina. Por último y de manera interesante, los autores reportaron una disminución en la expresión de una proteína que participa en la regulación de los ciclos fisiológicos de estos insectos: la hormona juvenil esterasa. Esta disminución prolongaría la duración de la etapa larvaria, probablemente por una disminución general del metabolismo, evidenciando un déficit energético derivado de la ingesta exclusiva de PE.

\section{ProyeCCIONES FUTURAS Y FACTIBILIDAd TÉCNICA- ECONÓMICA DE LOS SISTEMAS DE DEGRADACIÓN DE PLÁSTICOS POR LARVAS DE INSECTOS}

Derivado de lo expuesto en los párrafos anteriores, queda claro que la ingesta y consumo de PE y PS por distintos tipos de larvas de insectos es una realidad y algunas evidencias sugieren que estos tratamientos biológicos pueden provocar despolimerización e incluso mineralización de estos materiales. Por otro lado, debemos mencionar que, a pesar de existir estudios alentadores, la viabilidad técnica y económica, así como el impacto ambiental que la implementación de este tipo de procesos pueda tener a gran escala y a largo plazo continúa siendo objeto de debate. Dicho debate gira en torno a I) la evidencia contrastante sobre la biotransformación del PE, II) la baja capacidad que presentan las larvas para desarrollarse de manera óptima cuando se alimentan exclusivamente de polímeros plásticos y III) el bajo valor agregado de los productos de degradación del PE y el PS.

Billen, Khalifa, Van Gerven, Tavernier \& Spatari (2020) estudiaron los metabolitos generados durante la biodegradación de LDPE por larvas de G. mellonella. Sus 
resultados muestran una mínima formación de etilenglicol durante las primeras $36 \mathrm{~h}$ de contacto, por lo que los autores sugieren que la transformación química del polímero se debe más a efectos mecánicos de la masticación que a una degradación biológica, lo anterior concuerda con lo sugerido anteriormente por Weber, Pusch \& Opatz (2017) y discrepa de los resultados mencionados anteriormente en este texto. Esta evidencia contrastante podría atribuirse a que la capacidad para biotransformar PE posiblemente no sea una característica globalmente conservada en la especie. Aunado a lo anterior, se identificaron microplásticos de PE en el humus excretado, lo cual representaría un riesgo por la posible dispersión de estos contaminantes en el ambiente o su ingreso a las cadenas tróficas. Por otra parte, los autores realizaron un análisis de factibilidad de un sistema de tratamiento de PE a gran escala -el único reporte de este tipo, hasta donde tenemos conocimiento-, este análisis estima un requerimiento de entre 4 y 10 toneladas de $G$. mellonella para el tratamiento de una tonelada de LDPE, lo que se traduce en un gasto neto de entre $300 €$ y $1,000 €$ por tonelada de plástico, montos que exceden los costos de incineración para producción de energía en Europa (Ijgosse, 2019). Derivado de lo anterior, la utilización de estos organismos para el tratamiento de PE parece poco viable, especialmente ante la imposibilidad de utilizar la biomasa o el humus como productos de valor agregado debido a la presencia de microplásticos en los mismos. También es importante señalar que la utilización de larvas de la cera debería realizarse con especial cuidado y control, ya que es una plaga agresiva para las abejas que puede afectar significativamente a los ecosistemas.

En otro estudio se diseñó un modelo de balance energético dinámico para evaluar la factibilidad técnica de un sistema de tratamiento de EPS por larvas de T. molitor cultivadas con dietas a base de este polímero (Matyja, Rybak, Hanus-Lorenz, Wróbel \& Rutkowski, 2020). Los resultados mostraron que los organismos alimentados exclusivamente con EPS exhibieron una considerable pérdida de masa, un pobre crecimiento y una tasa de supervivencia de apenas $4 \%$ después de 98 días; en contraste, los organismos cultivados con una dieta convencional de avena y dietas mixtas de PS + avena mostraron un crecimiento y una tasa de supervivencia similar ( $\approx 50 \%$ ) en los 98 días de tratamiento. Lo anterior sugiere que el aporte energético que brinda el plástico a estos organismos es insuficiente para completar su ciclo de vida de manera óptima, por lo que el cultivo de estos insectos con una dieta exclusiva de PS sería insostenible a largo plazo. A pesar de que la cría de larvas de harina alimentadas exclusivamente con polímeros plásticos parece no ser una estrategia factible a largo plazo, los hallazgos reportados en los últimos años sugieren que enriquecer la dieta de estos organismos con sustratos convencionales (semillas, cereales, vegetales, etc.) promueve, en la mayoría de los casos, un mejor desarrollo de los mismos, por lo que esta estrategia se presenta como una opción interesante que debe estudiarse con mayor profundidad y focalizarse en la utilización de sustratos económicos y/o subproductos agroindustriales. Por otro lado, de comprobarse la ausencia de metabolitos tóxicos en las excretas o biomasa, se abriría la posibilidad de comercializar los sub-productos derivados de la cría de estos insectos, agregándole valor a una posible aplicación industrial que permitiría el desarrollo de una economía circular, por ejemplo, el humus de T. molitor es un potente bio-fertilizante (Houben, Daoulas, Faucon \& Dulaurent, 2020) y la misma biomasa de estos organismos, rica en proteínas y lípidos, se puede utilizar como alimento vivo para animales y peces, carnada para pesca, para la elaboración de alimentos fortificados o para la obtención de aceites (Grau, Vilcinskas \& Joop, 2017).

Con base en lo señalado, podemos concluir que el empleo de insectos para el tratamiento de polímeros plásticos no es actualmente una estrategia viable a gran escala; sin embargo, es importante señalar que la investigación en esta área no debería desincentivarse, ya que, considerando la gran diversidad de estas familias de insectos, parece probable que existan especies cuya capacidad para biodegradar estos polímeros aún no haya sido descubierta. Además, las técnicas moleculares modernas como la metagenómica, proteómica o metabolómica así como las técnicas de ingería genética actuales abren un abanico de oportunidades para la identificación y mejoramiento de las bacterias entéricas de estos organismos y para el aislamiento y producción heteróloga de proteínas que estén directamente involucradas en los procesos de biodegradación de estos compuestos.

\section{AGRADECIMIENTOS}

Los autores agradecen al Departamento de Ciencias Químico Biológicas de la Universidad Autónoma de Ciudad Juárez, por el apoyo para llevar a cabo las actividades de investigación.

\section{REFERENCIAS}

Aboelkheir, M. G., Visconte, L. Y., Oliveira, G. E., ToledoFilho, R. D. \& Souza, F. G. (2019). The biodegradative effect of Tenebrio molitor Linnaeus larvae on vulcanized SBR and tire crumb. Science of the Total Environment, 649, 1075-1082. DOI:10.1016/j.scitotenv.2018.08.228

Arthuz-López, L. \& Pérez-Mora, W. (2019). Alternativas de bajo impacto ambiental para el reciclaje del poliestireno expandido a nivel mundial. Informador Técnico, 83(2), 209-219. https://doi.org/10.23850/22565035.1638

Billen, P., Khalifa, L., Van Gerven, F., Tavernier, S. \& Spatari, S. (2020). Technological application potential of polyethylene and polystyrene biodegradation by macro-organisms such as mealworms and wax moth larvae. Science of the Total Environment, 735, 139521. DOI:10.1016/j.scitotenv.2020.139521

Bombelli, P., Howe C. J. \& Bertocchini, F. (2017). Polyethylene bio-degradation by caterpillars of the wax 
moth Galleria mellonella. Current Biology, 27, R283R293. DOI: 10.1016/j.cub.2017.02.060.

Brandon, A. M., Gao, S. H., Tian, R., Ning, D., Yang, S. S., Zhou, J., Wu, W. M. \& Criddle, C. S. (2018). Biodegradation of polyethylene and plastic mixtures in mealworms (Larvae of Tenebrio molitor) and effects on the gut microbiome. Environmental Science and Technology, 52(11), 6526-6533. DOI:10.1021/acs.est.8b02301

Bryning, G. P., Chambers, J. \& Wakefield, M. E. (2005). Identification of a sex pheromone from male yellow mealworm beetles, Tenebrio molitor. Journal of Chemical Ecology, 31(11), 2721-2730. DOI:10.1007/s10886-0057622-x

Cassone, B. J., Grove, H. C., Elebute, O., Villanueva, S. M. P. \& LeMoine, C. M. R. (2020). Role of the intestinal microbiome in low-density polyethylene degradation by caterpillar larvae of the greater wax moth, Galleria mellonella. Proccedings of the Royal Society B, 287, 20200112. DOI:10.1098/rspb.2020.0112

Cifuentes-Ruiz, P. \& Zaragoza-Caballero, S. (2014). Biodiversidad de Tenebrionidae (Insecta: Coleoptera) en México. Revista Mexicana de Biodiversidad Suplemento, 85, S325-S331. DOI: http://dx.doi.org/10.7550/ rmb. 31690

Eriksen, M., Lebreton, L. C. M., Carson, H. S., Thiel, M., Moore, C. J., Borrero, J. C., Galgani, F., Ryan, P. G. \& Reisser, J. (2014). Plastic pollution in the world's oceans: more than 5 trillion plastic pieces weighing over 250,000 tons afloat at sea. PLoS ONE, 9(12), e111913. DOI:10.1371/journal.pone.0111913

Fabreag, M. A. C. \& Familara, J. A. (2019). Biodegradation of expanded polystyrene (EPS) (Styrofoam) block as feedstock to Tribolium castaneum (Red Flour Beetle) imago: A promising plastic-degrading process. World News of Natural Sciences, 24, 145-156. EISSN 25435426

Geyer, R., Jambeck, J. R. \& Law, K. L. (2017). Production, use, and fate of all plastics ever made. Science advances, 3(7), e1700782 . DOI: 10.1126/sciadv.1700782

Grau, T., Vilcinskas, A. \& Joop, G. (2017). Sustainable farming of the mealworm Tenebrio molitor for the production of food and feed. Zeitschrift Fur Naturforschung - Section C Journal of Biosciences, 72(9-10), 337-349. https://doi. org/10.1515/znc-2017-0033

Hassan, M. W., Qasim, M. U., Iqbal, J. \& Jamil, M. (2014). Study of penetration ability by Tribolium castaneum (Herbst.) (Coleoptera: Tenebrionidae) through different loose plastic. Journal of Pure and Applied Sciences, 2433(1-2), 17-20. ISSN: 0255-3643

Hassan, M. W., Gulraize, Ali, U., Rehman, F. U., Najeeb, H., Sohail, M., Irsa, B., Muzaffar, Z. \& Chaudhry, M. S. (2016). Evaluation of standard loose plastic packaging for the management of Rhyzopertha dominica (F.) (Coleoptera: Bostrichidae) and Tribolium castaneum
(Herbst) (Coleoptera: Tenebrionidae). Journal of Insect Science, 16(1), 91. DOI:10.1093/jisesa/iew075

Houben, D., Daoulas, G., Faucon, M. P. \& Dulaurent, A. M. (2020). Potential use of mealworm frass as a fertilizer: Impact on crop growth and soil properties. Scientific Reports, 10(1), 1-9. https://doi.org/10.1038/s41598-02061765-X

Ijgosse, J. (2019). Incineración y población recicladora: Una guía técnica sobre aprovechamiento energético. Women In Informal Employment Globalizing and Organizing, WIEGO. 28 p.

Kim, H. R., Lee, H. M., Jeon, E., Yu, H. C., Jeon, E., Lee, S., Li, J. \& Kim, D. H. (2020). Biodegradation of polystyrene by Pseudomonas sp. isolated from the gut of superworms. Environmental Science \& Technology, 54(11), 69876996. DOI: 10.1021 acs.est.0c01495

Kong, H. G., Kim, H. H., Chung, J. H. Jun, J., Lee, S., Kim, H. M., Jeon, S., Park, S. G., Bhak, J. \& Ryu, C. M. (2019). The Galleria mellonella hologenome supports microbiotaindependent metabolism of long-chain hydrocarbon beeswax. Cell Reports, 26, 2451-2464. DOI: 10.1016/j. celrep.2019.02.018

Kundungal, H., Gangarapu, M., Sarangapani, S., Patchaiyappan, A. \& Purushothaman, S.D. (2019 a). Efficient biodegradation of polyethylene (HDPE) waste by the plastic-eating lesser waxworm (Achroia grisella). Environmental Science and Pollution Research, 26, 18509-18519. DOI: 10.1007/s11356-019-05038-9

Kundungal,H., Gangarapu,M.,Sarangapani,S.,Patchaiyappan, A. \& Purushothaman, S. D. (2019 b). Role of pretreatment and evidence for the enhanced biodegradation and mineralization of low density polyethylene films by greater waxworm. Environmental Technology, 42(5), 717-730. DOI: 10.1080/09593330.2019.1643925

Leung, D., Yang, D., Li, Z., Zhao, Z., Chen, J. \& Zhu, L. (2012). Biodiesel from Zophobas morio larva oil: process optimization and FAME characterization. Industrial and Engineering Chemistry Research, 51(2), 1036-1040. DOI:10.1021/ie201403r

Lou, Y., Ekaterina, P., Yang, S., Lu, B., Liu, B. F. Ren, N., Corvini, P. \& Xing, D. (2020). Bio-degradation of polyethylene and polystyrene by greater wax moth larvae (Galleria mellonella L.) and the effect of codiet supplementation on the core gut microbiome. Environmental Science \& Technology, 54 (5), 2821-2831. DOI: 10.1021/acs.est.9b07044

Maharana T, Negi Y. S. \& Mohanty B. (2007). Recycling of polystyrene. Polymer-. Plastics Technology and Engineering, 46 (7), 729-736. doi:10.1080/03602550701273963

Matyja, K., Rybak, J., Hanus-Lorenz, B., Wróbel, M. \& Rutkowski, R. (2020). Effects of polystyrene diet on Tenebrio molitor larval growth, development and survival: Dynamic Energy Budget (DEB) model analysis. 
Environmental Pollution, 264, 114740. DOI:10.1016/j. envpol.2020.114740

Nukmal, N., Umar, S., Amanda, S. P. \& Kanedi, M. (2018). Effect of styrofoam waste feeds on the growth, development and fecundity of mealworms (Tenebrio molitor). OnLine Journal of Biological Sciences, 18(1), 24-28. DOI:10.3844/ojbsci.2018.24.28

ONU, Organización de las Naciones Unidas. (2020). La marea de plástico causada por el COVID-19 también es un peligro para la economía y la naturaleza. https://news. un.org/es/story/2020/07/1478011

Peng, B. Y., Su, Y., Chen, Z., Chen, J., Zhou, X., Benbow, M. E., Criddle, C. S., Wu, W. M. \& Zhang, Y. (2019). Biodegradation of polystyrene by dark (Tenebrio obscurus) and yellow (Tenebrio molitor) mealworms (Coleoptera: Tenebrionidae). Environmental Science and Technology, 53(9), 5256-5265. DOI:10.1021/acs. est.8b06963

Peng, B. Y., Li, Y., Fan, R., Chen, Z., Chen, J., Brandon, A. M., Criddle, C. S., Zhang, Y. \& Wu, W. M. (2020). Biodegradation of low-density polyethylene and polystyrene in superworms, larvae of Zophobas atratus (Coleoptera: Tenebrionidae): Broad and limited extent depolymerization. Environmental Pollution, 266, 115206. DOI:10.1016/j.envpol.2020.115206

Peydaei, A., Bagheri, H., Gurevich, L., de Jonge, N. \& Nielsen, J. L. (2020). Impact of polyethylene on salivary glands proteome in Galleria mellonella. Comparative Biochemistry and Physiology - Part D: Genomics and Proteomics, 34, 100678. DOI: 10.1016/j. cbd.2020.100678

Przemieniecki, S. W., Kosewska, A., Ciesielski, S. \& Kosewska, O. (2020). Changes in the gut microbiome and enzymatic profile of Tenebrio molitor larvae biodegrading cellulose, polyethylene and polystyrene waste. Environmental Pollution, 256, 113265. DOI:10.1016/j. envpol.2019.113265

Vázquez-Morillas, A., Sotelo-Navarro, P. X., EspinozaValdemar, R. M., Velasco-Pérez, M., Quecholac-Piña, X., Beltrán-Villavicencio, M. \& Álvarez-Zeferino, J. C. (2018). Degradación \& Biodegradación de plásticos. Asociacion Nacional de Industria Química, A.C., 23.

Wang, Z., Xin, X., Shi, X. \& Zhang, Y. (2020). A polystyrenedegrading Acinetobacter bacterium isolated from the larvae of Tribolium castaneum. Science of the Total Environment, 726, 138564. DOI:10.1016/j. scitotenv.2020.138564

Weber, C., Pusch, S. \& Opatz, T. (2017). Polyethylene biodegradation by caterpillars? Current Biology, 27(15), R744-R745. https://doi.org/10.1016/j.cub.2017.07.004
Wu, Q., Tao, H. \& Wong, M. H. (2019). Feeding and metabolism effects of three common microplastics on Tenebrio molitor L. Environmental Geochemistry and Health, 41(1), 17-26. DOI:10.1007/s10653-018-0161-5

Xia, M., Wang, J., Huo, Y. X. \& Yang, Y. (2020). Mixta tenebrionis sp. Nov., isolated from the gut of the plasticeating mealworm Tenebrio molitor L. International Journal of Systematic and Evolutionary Microbiology, 70(2), 790-796. DOI:10.1099/ijsem.0.003826

Yang, J., Yang, Y., Wu, W. M., Zhao, J. \& Jiang, L. (2014). Evidence of polyethylene biodegradation by bacterial strains from the guts of plastic-eating waxworms. Environmental Science and Technology, 48, 13776-13784. DOI: 10.1021/es504038a

Yang, Y., Yang, J., Wu, W. M., Zhao, J., Song, Y., Gao, L., Yang, R. \& Jiang, L. (2015 a). Biodegradation and mineralization of polystyrene by plastic-eating mealworms: part 1 . chemical and physical characterization and isotopic tests. Environmental Science and Technology, 49(20), 1208012086. DOI:10.1021/acs.est.5b02661

Yang, Y., Yang, J., Wu, W. M., Zhao, J., Song, Y., Gao, L., Yang, R. \& Jiang, L. (2015 b). Biodegradation and mineralization of polystyrene by plastic-eating mealworms: part 2. role of gut microorganisms. Environmental Science and Technology, 49(20), 12087-12093. DOI:10.1021/acs. est. 5 b02663

Yang, S. S., Brandon, A. M., Andrew Flanagan, J. C., Yang, J., Ning, D., Cai, S. Y., Fan, H. Q., Wang, Z. Y., Ren, J., Benbow, E., Ren, N. Q., Waymouth, R. M., Zhou, J., Criddle, C. S. \& Wu, W. M. (2018 a). Biodegradation of polystyrene wastes in yellow mealworms (larvae of Tenebrio molitor Linnaeus): Factors affecting biodegradation rates and the ability of polystyrene-fed larvae to complete their life cycle. Chemosphere, 191, 979-989. DOI:10.1016/j.chemosphere.2017.10.117

Yang, S. S., Wu, W. M., Brandon, A. M., Fan, H. Q., Receveur, J. P., Li, Y., Wang, Z. Y., Fan, R., McClellan, R. L., Gao, S. H., Ning, D., Phillips, D. H., Peng, B. Y., Wang, H., Cai, S. Y., Li, P., Cai, W. W., Ding, L. Y., Yang, J., Zheng, M., Ren, J., Zhang, Y. L., Gao, J., Xing, D., Ren, N. Q., Waymouth, R. M., Zhou, J., Tao, H. C., Picard, C. J., Benbow, M. E. \& Criddle, C. S. (2018 b). Ubiquity of polystyrene digestion and biodegradation within yellow mealworms, larvae of Tenebrio molitor Linnaeus (Coleoptera: Tenebrionidae). Chemosphere, 212, 262271. DOI:10.1016/j.chemosphere.2018.08.078

Yang, Y., Wang, J. \& Xia, M. (2020). Biodegradation and mineralization ofpolystyrenebyplastic-eating superworms Zophobas atratus. Science of the Total Environment, 708, 135233. DOI:10.1016/j.scitotenv.2019.135233 